\title{
DENSIDADE DE FÊMEAS DE PALMISTICHUS ELAEISIS DELVARE \& LASALLE, 1993 (HYMENOPTERA: EULOPHIDAE) PARA SUA REPRODUÇÃO EM PUPAS DE ANTICARSIA GEMMATALIS HÜBNER, 1818 (LEPIDOPTERA: NOCTUIDAE)
}

\author{
P.L. Pastori ${ }^{1}$, F.F. Pereira' ${ }^{2}$, J.C. Zanuncio ${ }^{3}$, H.N. de Oliveira ${ }^{4}$, V.F.R. Calado ${ }^{2}$, R.O. Silva ${ }^{3}$ \\ ${ }^{1}$ Universidade Federal do Ceará, Faculdade de Fitotecnia, Av. Mister Hull, 2977, CEP 60356-000, Fortaleza, \\ CE, Brasil. E-mail: plpastori@ufc.br
}

\begin{abstract}
RESUMO
A densidade de parasitoides por hospedeiro influencia nas suas principais características biológicas. Assim, o objetivo desse trabalho foi avaliar o desenvolvimento do parasitoide Palmistichus elaeisis Delvare \& LaSalle, 1993 (Hymenoptera: Eulophidae) em pupas de Anticarsia gemmatalis Hübner, 1818 (Lepidoptera: Noctuidae) expostas nas densidades de 1:1, 2:1, 4:1, 6:1, 8:1, 10:1 ou 12:1 parasitoides/hospedeiro, respectivamente. Os parasitoides permaneceram em contato com as pupas por 24 horas em tubos de vidro $(14,0 \times 2,2 \mathrm{~cm})$, acondicionadas em câmara climatizada a $25 \pm 1^{\circ} \mathrm{C}, 70 \pm 10 \%$ de UR e fotofase de 14 horas. Observou-se 54,54 e 90,90\% de pupas parasitadas nas densidades de 1:1 e 2:1, respectivamente, e 100,00\% nas demais densidades com melhor índice de emergência $(72,72 \%$ ) na densidade 6:1. A duração do ciclo de vida (ovo-adulto) decresceu com o aumento da densidade de fêmeas e variou de 20 a 22 dias. A progênie variou de 16 a 225 descendentes por pupa, com melhor resultado na densidade 8:1. A razão sexual do parasitoide variou de 0,95 $\pm 0,00$ a $0,97 \pm 0,01$, sem diferença significativa e o comprimento do corpo de fêmeas e de machos variou de 1,95 $\pm 0,02$ a 2,22 $\pm 0,03 \mathrm{~mm}$ e de 1,49 $\pm 0,02$ a 1,87 $\pm 0,01 \mathrm{~mm}$, respectivamente. Seis a oito fêmeas de $P$. elaeisis por pupa de $A$. gemmatalis proporcionam maior emergência e progênie do parasitoide, viabilizando sua criação massal.
\end{abstract}

PALAVRAS-CHAVE: Hospedeiro alternativo, criação massal, taxa de parasitismo, parasitoides.

\begin{abstract}
DENSITY OF FEMALES OF PALMISTICHUS ELAEISIS DELVARE \& LASALLE, 1993 (HYMENOPTERA: EULOPHIDAE) FOR REPRODUCTION IN ANTICARSIA GEMMATALIS HÜBNER, 1818 (LEPIDOPTERA: NOCTUIDAE) PUPAE. The density of parasitoids per host affects their biological characteristics. The present study aimed to evaluate the development of Palmistichus elaeisis Delvare \& LaSalle, 1993 (Hymenoptera: Eulophidae) in Anticarsia gemmatalis Hübner, 1818 (Lepidoptera: Noctuidae) pupae exposed to 1:1, 2:1, 4:1, 6:1, 8:1, 10:1 or 12:1 females/ host, respectively. The parasitoid remained in contact with pupae for $24 \mathrm{~h}$ in glass tubes $(14.0 \mathrm{x}$ $2.2 \mathrm{~cm}$ ), packed in a climate controlled chamber regulated at $25 \pm 1^{\circ} \mathrm{C}, 70 \pm 10 \%$ relative humidity and photophase of 14 hours. We observed 54.54 and $90.90 \%$ of pupae parasitized at densities of 1:1 and $2: 1$, respectively, and $100.00 \%$ in the other densities with a better emergence index $(72.72 \%)$ at a density of $6: 1$. The duration of the (egg-adult) life cycle decreased with increasing density of females and varied from 20 to 22 days. The offspring varied from 16 to 225 descendants per $A$. gemmatalis pupae with best results at the density 8:1. The sex ratio of the parasitoid varied from $0.95 \pm 0.00$ to $0.97 \pm 0.01$ without significant differences, and the body length of females and of males varied from $1.95 \pm 0.02$ to $2.22 \pm 0.03 \mathrm{~mm}$ and $1.49 \pm 0.02$ to $1.87 \pm 0.01 \mathrm{~mm}$, respectively. Six to eight of $P$. elaeisis females per $A$. gemmatalis pupae provide more emergence and offspring of the parasitoid, allowing for its mass rearing.
\end{abstract}

KEY WORDS: Alternative host, mass rearing, parasitism rate, parasitoids.

${ }^{2}$ Universidade Federal da Grande Dourados, Faculdade de Ciências Biológicas e Ambientais, Dourados, MS, Brasil. ${ }^{3}$ Universidade Federal de Viçosa, Departamento de Biologia Animal, Viçosa, MG, Brasil.

${ }^{4}$ Embrapa Agropecuária Oeste, Laboratório de Entomologia, Dourados, MS, Brasil. 


\section{INTRODUÇÃO}

A incidência de insetos-praga é um dos fatores ambientais que regulam a produtividade das florestas plantadas (BITTENCOURT; BeRTI FILHO, 2004; ZANUNCIO et al., 2009). A extensão dos plantios, a altura das árvores e o comportamento de alguns insetos-praga reduzem a eficiência da pulverização de produtos fitossanitários (BITTENCOURT; BERTI FilHo, 2004; ZANUNCio et al., 2010). Assim, métodos alternativos ao controle químico e que favoreçam a conservação e o uso sustentável dos recursos naturais têm sido propostos (BARBOSA et al., 2008) e, dentre eles, a utilização de inimigos naturais apresenta características adequadas por integrar o manejo de pragas (BARBOSA et al., 2008) e baixos custos relativos (MONTEIRO et al., 2006).

Palmistichus elaeisis Delvare \& LaSalle, 1993 (Hymenoptera: Eulophidae) foi registrado em pupas de Eupseudosoma involuta (Sepp, 1852), Euselasia eucerus Hewitson, 1872 (Lepidoptera: Riodinidae) (Delvare; LaSalle, 1993), Sabulodes sp. (Lepidoptera: Geometridae) (BitTencourt; Berti Filho, 2004a), Thyrinteina arnobia (Stoll, 1782) e Thyrinteina leucoceraea Rindge, 1961 (Lepidoptera: Geometridae) em plantios de eucalipto no Brasil (Pereira et al., 2008). $P$. elaeisis é um endoparasitoide gregário, polífago, idiobionte e inviabiliza pupas de Lepidoptera (Pereira et al., 2008) e pode representar uma alternativa para o controle de lagartas desfolhadoras de eucalipto (Pereira; Zanuncio, 2005).

No entanto, para a utilização efetiva de um agente de controle biológico, uma etapa importante é a seleção de um hospedeiro alternativo que seja reconhecido e fisiologicamente adequado (VINSON; IWANTSCH, 1980). Além disso, o hospedeiro deve ser produzido com facilidade, de maneira eficiente e econômica (PARra et al., 2002), e aspectos como qualidade nutricional, tamanho, idade, resistência do tegumento e capacidade de resposta imunológica aos parasitoides (GODFRAY, 1994) e sexo (BITTENCOURT; BERTIFILHO, 1999) devem ser considerados na seleção do hospedeiro alternativo.

O desenvolvimento de metodologias de criação massal depende do conhecimento de aspectos bióticos como maturidade e razão sexual, tipo de reprodução, longevidade, duração do ciclo de vida (ovo-adulto), especificidade, discriminação hospedeira, capacidade de parasitismo, além da sensibilidade dos parasitoides a fatores abióticos como temperatura, luz e umidade (GODFraY, 1994; BitTenCourt et al., 2004; Gunduz; Gulel, 2005).

A densidade de parasitoides por hospedeiro pode afetar a produção de descendentes (PEREIRA et al., 2010b), a razão sexual da prole (Сно et al., 2001), a capacidade de parasitismo (SAMPAIO et al., 2001), a duração do ciclo, o tamanho do corpo e a lon- gevidade de parasitoides (Silva-Torres; Matthews, 2003). Isso indica a necessidade de se aprimorar as metodologias de criação massal de parasitoides em laboratório visando conhecer, principalmente, a densidade ideal em relação ao hospedeiro para se minimizar o custo com mão de obra e maximizar a produção (SAGARRA et al., 2000).

Palmistichus elaeisis se reproduz satisfatoriamente em pupas de Anticarsia gemmatalis Hübner, 1818 (Lepidoptera: Noctuidae) (BItTencourt; Berti Filho, 2004a; BITTENCOURT et al., 2004) eessa, por sua vez, pode ser facilmente criada em dieta artificial (GREENE et al., 1976). No entanto, não é conhecido o número ideal de fêmeas de $P$. elaeisis para produzir progênie nesse hospedeiro. Como P. elaeisis possui hábito gregário, torna-se necessário determinar a densidade ideal de fêmeas desse parasitoide por pupa hospedeira para obter melhor produção de descendentes em laboratório (Pereira et al., 2010b). Assim, o objetivo foi determinar o desenvolvimento de $P$. elaeisis em pupas de A. gemmatalis expostos ao parasitismo em diferentes densidades parasitoides/ hospedeiro.

\section{MATERIAL E MÉTODOS}

O experimento foi realizado no Laboratório de Controle Biológico de Insetos (LCBI) do Instituto de Biotecnologia Aplicada à Agropecuária (BIOAGRO) da Universidade Federal de Viçosa (UFV), em Viçosa, Minas Gerais, com as etapas:

Criação de A. gemmatalis. Ovos provenientes da criação estoque, mantida em dieta artificial, no LCBI/ BIOAGRO/UFV, foram colocados sobre papel filtro umedecido no interior de placas de Petri $(10,0$ x 2,5 $\mathrm{cm})$ e, as lagartas recém-eclodidas transferidas com pincel fino para recipientes plásticos onde receberam diariamente dieta artificial (GRENNE et al., 1976). Os recipientes com as lagartas foram mantidos em sala climatizada a $25 \pm 2^{\circ} \mathrm{C}, 70 \pm 10 \%$ de umidade relativa (UR) e fotofase de 14 horas, até a fase de pupa. As pupas de A. gemmatalis foram colocadas em gaiolas teladas $(33 \times 33 \times 33 \mathrm{~cm})$, revestidas internamente nas laterais com papel tipo sulfite, utilizado como substrato para oviposição. Os adultos foram alimentados com solução aquosa de mel a $10 \%$.

Criação do parasitoide. Adultos recém-emergidos de P. elaeisis foram mantidos em tubos de vidro $(14,0 \times 2,2 \mathrm{~cm})$ tampados com algodão e alimentados com gotículas de mel. Pupas de A. gemmatalis, com até 48 horas deidade, foram expostas ao parasitismo, por 24 horas a $25 \pm 2^{\circ} \mathrm{C}$, umidade relativa de $70 \pm$ $10 \%$ e fotofase de 14 horas para manutenção da criação de P. elaeisis.

Procedimento experimental. Fêmeas de P. elaeisis, com 72 horas de idade foram colocadas em tubos de vidro $(14,0 \times 2,2 \mathrm{~cm})$, com alimento (gotículas de 
mel) e expostas a pupas de $A$. gemmatalis (peso de $195,94 \pm 6,02 \mathrm{mg}$ ), com 24-48 horas de idade, por 24 horas, nas densidades de 1:1, 2:1, 4:1, 6:1, 8:1, 10:1 ou 12:1, parasitoides/hospedeiro, respectivamente. O experimento foi mantido em câmara climatizada a $25 \pm 1^{\circ} \mathrm{C}, 70 \pm 10 \%$ de umidade relativa e fotofase de 14 horas. Após 24 horas, as fêmeas de P. elaeisis foram retiradas dos tubos e esses, contendo as pupas parasitadas, foram mantidas na mesma câmara até a emergência da progênie.

Os parâmetros avaliados foram: duração do ciclo de vida (ovo-adulto); porcentagem de parasitismo; porcentagem de emergência da progênie; número e tamanho dos parasitoides emergidos por pupa de A. gemmatalis, parâmetro esse avaliado com auxílio de ocular micrométrica acoplada ao microscópio estereoscópio e a razão sexual calculada pela equação: (RS $=\mathrm{n}^{\circ}$ de fêmeas $/ \mathrm{n}^{\circ}$ de adultos). O sexo dos indivíduos foi determinado com base nas características morfológicas da antena e do abdome (DeLVARE; LASALLE, 1993).

O delineamento experimental foi o inteiramente casualizado, sendo os tratamentos representados pelas densidades do parasitoide com doze repetições, cada uma, constituída por uma pupa de A. gemmatalis com respectiva densidade de fêmeas do parasitoide. Os dados da duração do ciclo e o número de parasitoides emergidos por pupa de A.gemmatalisforam submetidos à análise devariância (ANOVA) eà análise deregressão $(\mathrm{p} \leq 0,05)$. As porcentagens deparasitismoedeemergência de $P$. elaeisis foram submetidas ao teste binomial ( $\mathrm{p} \leq$ 0,05). Os dados da razão sexual e do tamanho do corpo de fêmeas e machos de $P$. elaeisis foram submetidos à análise de variância (ANOVA) eao teste deScott-Knott $(\mathrm{p} \leq 0,05)$.

\section{RESULTADOS}

O parasitismo de $P$. elaeisis em pupas de $A$. gemmatalis foi alterado pela densidade de fêmeas, com $54,54 \%$ e $90,90 \%$ de pupas parasitadas nas densidades 1:1 e 2:1, respectivamente, e 100,00\% nas demais densidades $(p=0,0278)$ (Fig. 1). A emergência de indivíduos de P. elaeisis de pupas de A. gemmatalis apresentou melhor resultado na densidade 6:1, 72,72\% (Fig. 1).

A duração do ciclo de vida (ovo-adulto) variou de 20 a 22 dias e decresceu com o aumento da densidade de fêmeas de $P$. elaeisis $(F=8,9195 ; \mathrm{p}=$ 0,0012; $\mathrm{gl}_{\text {erro }}=27$ ) (Fig. 2). A progênie de P. elaeisis foi alterada pela densidade de fêmeas desse parasitoide variando de 16 a 225 descendentes por pupa e ponto de máxima na densidade 7,8 parasitoides/pupa $\left(\mathrm{F}=10,33 ; \mathrm{p}=0,0005 ; \mathrm{gl}_{\text {erro }}=\right.$ 27) (Fig. 3). A progênie por fêmea de $P$. elaeisis diferiu entre tratamentos e reduziu com aumento da densidade desse parasitoide $(\mathrm{F}=12,6625 ; \mathrm{p}=$ 0,0002; $\mathrm{gl}_{\text {erro }}=27$ ) (Fig. 4).

A razão sexual de $P$. elaeisis foi semelhante entre tratamentos e variou de $0,95 \pm 0,01$ a $0,97 \pm$ $0,01(\mathrm{~F}=2,12 ; \mathrm{p}=0,25306)$ (Tabela 1). O tamanho do corpo de fêmeas e de machos (cabeça à extremidade abdominal) de $P$. elaeisis variou de 1,95 $\pm 0,02$ a $2,20 \pm 0,03 \mathrm{~mm}(\mathrm{~F}=20,479 ; \mathrm{p}=0,00000)$ e de 1,50 $\pm 0,02$ a $1,87 \pm 0,01 \mathrm{~mm}(\mathrm{~F}=12,499 ; \mathrm{p}=$ $0,00000)$, respectivamente. A largura da cápsula cefálica de fêmeas e machos variou de $0,61 \pm 0,00$ a $0,50 \pm 0,00 \mathrm{~mm}(\mathrm{~F}=57,425 ; \mathrm{p}=0,00000)$ e de 0,38 $\pm 0,01$ a $0,44 \pm 0,01 \mathrm{~mm}(\mathrm{~F}=5.200 ; \mathrm{p}=0,00042)$, respectivamente, com diferentes densidades do parasitoide (Tabela 1).

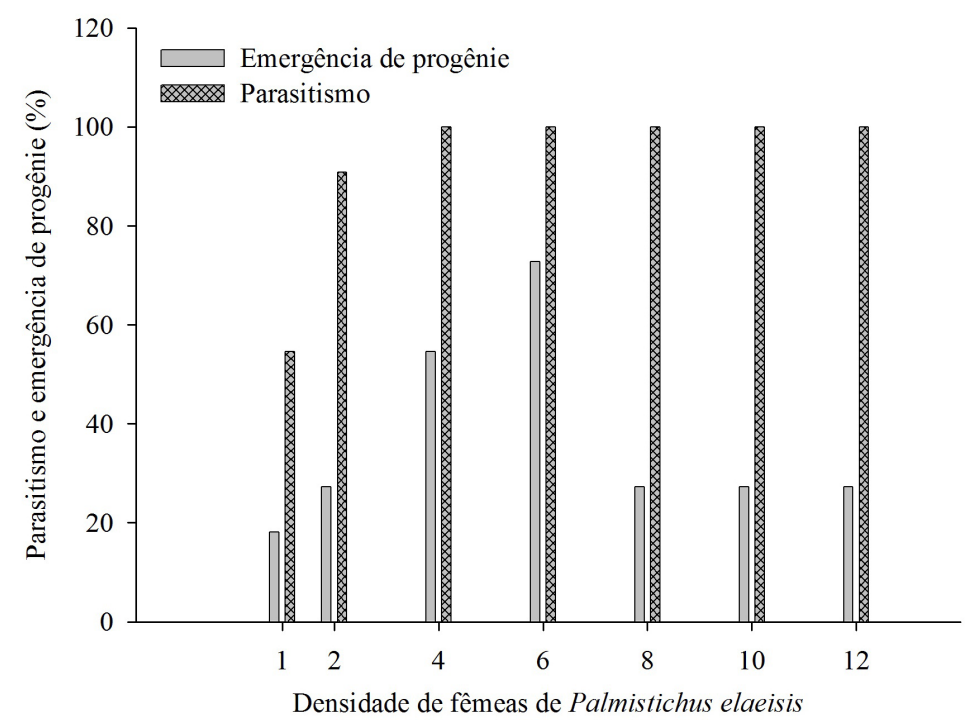

Fig. 1 - Parasitismo (\%) ( $p=00000)$ e emergência $(\%)(p=0,901)$ de Palmistichus elaeisis (Hymenoptera: Eulophidae) com variação na densidade de fêmeas por pupa de Anticarsia gemmatalis (Lepidoptera: Noctuidae). $25 \pm 2^{\circ} \mathrm{C}, 70 \pm 10 \%$ de umidade relativa e fotofase de 14 horas. 
Tabela 1 - Razão sexual, tamanho do corpo e da cápsula cefálica (média \pm erro padrão) ${ }^{1}$ de Palmistichus elaeisis (Hymenoptera: Eulophidae) com variação de densidade de fêmeas desse parasitoide por pupa de Anticarsia gemmatalis (Lepidoptera: Noctuidae). $25 \pm 2^{\circ} \mathrm{C}, 70 \pm 10 \%$ de umidade relativa e fotofase de 14 horas.

\begin{tabular}{|c|c|c|c|c|c|}
\hline Densidades & Razão sexual & \multicolumn{2}{|c|}{ Tamanho do corpo (mm) } & \multicolumn{2}{|c|}{ Cápsula cefálica (mm) } \\
\hline Parasitoide/pupa & {$[q /(\hat{0}+q)]$} & q & $\hat{\jmath}$ & q & $\hat{o}$ \\
\hline $1: 1$ & $0,95 \pm 0,01 \mathrm{a}$ & $2,20 \pm 0,03 a$ & $1,87 \pm 0,01 \mathrm{a}$ & $0,61 \pm 0,00 \mathrm{a}$ & $0,47 \pm 0,03 \mathrm{a}$ \\
\hline $2: 1$ & $0,96 \pm 0,01 \mathrm{a}$ & $2,22 \pm 0,03 a$ & $1,76 \pm 0,05 a$ & $0,61 \pm 0,00 \mathrm{a}$ & $0,50 \pm 0,00 \mathrm{a}$ \\
\hline $4: 1$ & $0,97 \pm 0,00 \mathrm{a}$ & $2,07 \pm 0,02 c$ & $1,65 \pm 0,04 \mathrm{a}$ & $0,58 \pm 0,01 b$ & $0,47 \pm 0,00 \mathrm{a}$ \\
\hline $6: 1$ & $0,97 \pm 0,01 \mathrm{a}$ & $1,97 \pm 0,03 d$ & $1,49 \pm 0,02 b$ & $0,52 \pm 0,01 d$ & $0,45 \pm 0,01 \mathrm{a}$ \\
\hline $8: 1$ & $0,96 \pm 0,02 \mathrm{a}$ & $1,95 \pm 0,02 d$ & $1,50 \pm 0,02 b$ & $0,50 \pm 0,00 d$ & $0,42 \pm 0,01 a$ \\
\hline $10: 1$ & $0,95 \pm 0,00 \mathrm{a}$ & $2,13 \pm 0,01 b$ & $1,74 \pm 0,01 \mathrm{a}$ & $0,60 \pm 0,00 \mathrm{a}$ & $0,46 \pm 0,00 \mathrm{a}$ \\
\hline $12: 1$ & $0,96 \pm 0,01 \mathrm{a}$ & $2,00 \pm 0,01 d$ & $1,72 \pm 0,05 \mathrm{a}$ & $0,55 \pm 0,01 \mathrm{c}$ & $0,43 \pm 0,03 a$ \\
\hline
\end{tabular}

${ }^{1}$ Médias seguidas de mesma letra minúscula, na coluna, não diferem entre si, pelo teste de Scott-Knott, ao nível de 5\% de probabilidade.

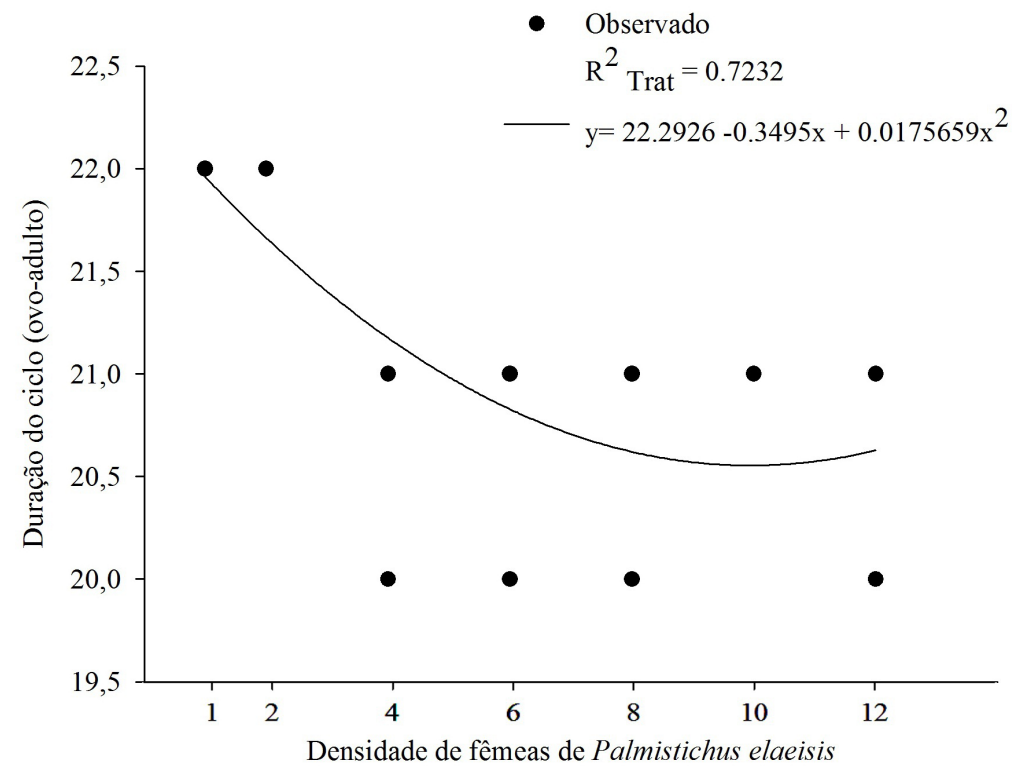

Fig. 2 - Duração do ciclo de vida (dias) (ovo-adulto) de Palmistichus elaeisis (Hymenoptera: Eulophidae) com variação na densidade de fêmeas por pupa de Anticarsia gemmatalis (Lepidoptera: Noctuidae). $25 \pm 2{ }^{\circ} \mathrm{C}, 70 \pm 10 \%$ de umidade relativa e fotofase de 14 horas.

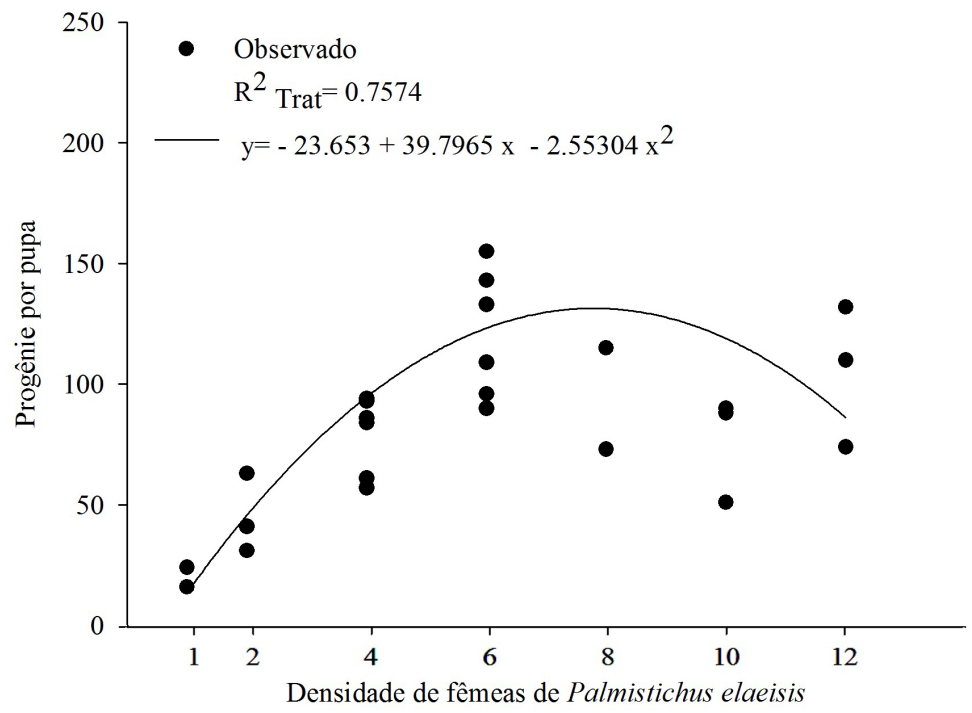

Fig. 3 - Progênie de Palmistichus elaeisis (Hymenoptera: Eulophidae) com variação na densidade de fêmeas desse parasitoide por pupa de Anticarsia gemmatalis (Lepidoptera: Noctuidae). $25 \pm 2^{\circ} \mathrm{C}, 70 \pm 10 \%$ de umidade relativa e fotofase de 14 horas. 


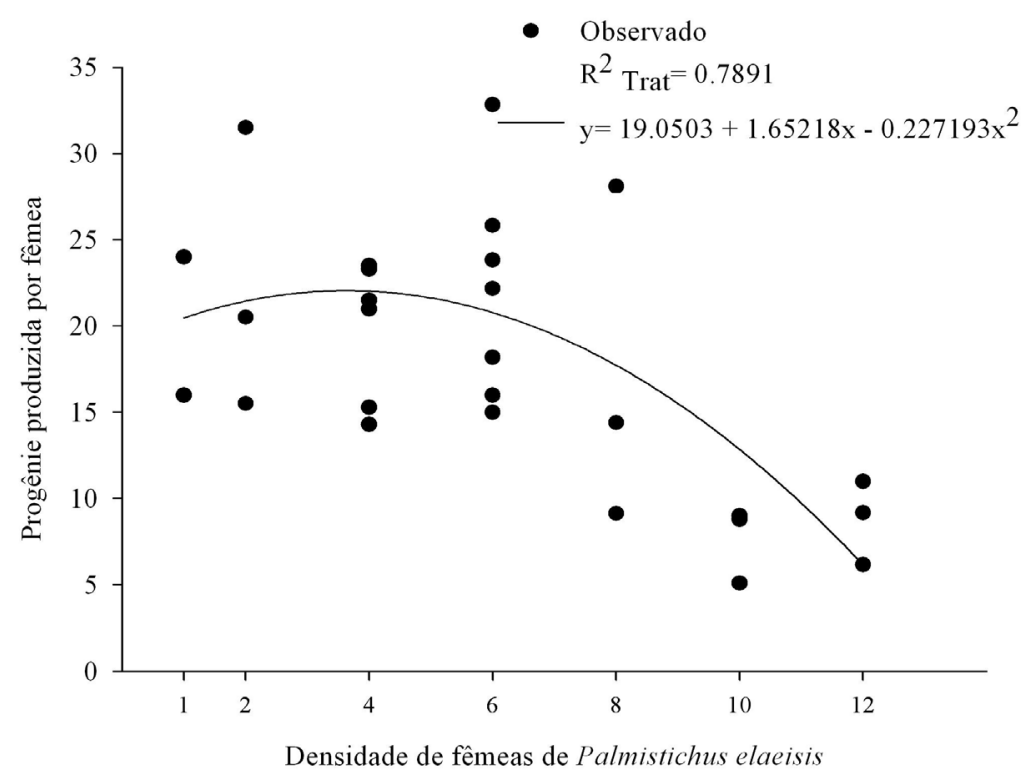

Fig. 4 - Progênie por fêmea de Palmistichus elaeisis (Hymenoptera: Eulophidae) com variação na densidade de fêmeas desse parasitoide por pupa de Anticarsia gemmatalis (Lepidoptera: Noctuidae). $25 \pm 2^{\circ} \mathrm{C}, 70 \pm 10 \%$ de umidade relativa e fotofase de 14 horas.

\section{DISCUSSÃO}

A densidade 6:1 foi a mais adequada para a emergência da progênie e isso pode estar relacionado ao fato de que proporções adequadas do parasitoide contribuem com a postura e a injeção de toxinas para reduzir a resposta imune do hospedeiro (SCHMID-HEMPEL, 2005), esse fato justifica também a variação na porcentagem de parasitismo, pois, provavelmente, a quantidade de ovos ou toxinas liberadas pelas fêmeas parasitoides em densidades inadequadas (1:1 e 2:1) não foram suficiente para neutralizar a ação imunológica do hospedeiro. As alterações na defesa celular e capacidade de encapsulamento de pupas de A. gemmatalis contra adultos dos parasitoides P. elaeisis e Trichospilus diatraeae Cherian \& Margabandhu, 1942 (Hymenoptera: Eulophidae) nas densidades de $1,3,5,7,9,11$ ou 13 parasitoides/ pupa estão relacionadas com a redução do número total de hemócitos na hemolinfa e a taxa de encapsulamento de pupas parasitadas. Nesse sentido, a redução da capacidade defensiva de A. gemmatalis está relacionada com o ajuste da densidade desses parasitoides para o seu desenvolvimento neste hospedeiro (ANDRADE et al., 2010). A emergência da progênie de $P$. elaeisis foi semelhante a observada em pupas de Bombyx mori Linneaus, 1758 (Lepidoptera: Bombycidae) (PEREIRA et al., 2010b) e para Anagyrus kamali Moursi, 1948 (Hymenoptera: Encyrtidae) em Maconellicoccus hirsutus Green, 1908 (Hemiptera: Pseudococcidae), ou seja, houve tendência ao aumento, seguido de queda da emergência das progênies com a maior densidade de parasitoides evidenciando a interfe- rência mútua entre fêmeas desses inimigos naturais (SAGARRA et al., 2000).

A capacidade reprodutiva de $P$. elaeisis em pupas de $A$. gemmatalis foi influenciada pela densidade de fêmeas parasitoides, semelhante ao observado para B. mori (PEREIRA et al., 2010b) e outros hospedeiros (Bittencourt; Berti Filho, 2006). Diferiu, entretanto. de $T$. diatraeae em pupas de Diatraea saccharalis (Fabricius, 1974), A. gemmatalis, Heliothis virescens (Fabricius, 1781) e Spodoptera frugiperda (Smith, 1797) (Lepidoptera: Noctuidae) expostas, individualmente, a uma ou várias fêmeas desse parasitoide, que quase duplicou e houve $100 \%$ de parasitismo com maior densidade de fêmeas desse parasitoide (PARON; BERTI-FILHO, 2000).

A redução da duração do ciclo de vida (ovoadulto) de P. elaeisis com o aumento do número de fêmeas indica que a competição dos imaturos desse parasitoide por nutrientes reduziu o tempo de desenvolvimento. Isso também foi observado expondo cinco densidades de Melittobia digitata Dahms, 1984 (Hymenoptera: Eulophidae) por pupa de Neobellieria bullata Parker, 1916 (Diptera: Sarcophagidae) (Silva-Torres; Matthews, 2003). A duração das fases imaturas de $P$. elaeisis em A. gemmatalis, D. saccharalis, S. frugiperda, T. arnobia e H. virescens foi de 18,9; 19,5; 19,7; 20,2 e 22 dias, respectivamente (BITTENCOURT; BERTI FILHO, 2004). Isto comprova que, além da densidade, o período do desenvolvimento desse parasitoide pode ser afetado pela espécie hospedeira (PAstori et al., 2008), pelo sexo (BitTencourt; Berti FILHO, 1999) ou pelas condições em que são armazenadas (PEREIRA et al., 2009). Esse fato, provavelmente, é 
justificado pela diferença de qualidade nutricional e tamanho das pupas, pois, após o parasitismo, o hospedeiro passa ser a fonte de alimento e o abrigo do endoparasitoide que, quando inadequado, altera seu desenvolvimento (Cônsoli; VINSON, 2002).

O número de indivíduos de $P$. elaeisis por pupa de A. gemmatalis foi maior na faixa de 6:1 a 10:1. Provavelmente, seis fêmeas desse parasitoide foram suficientes para neutralizar a atuação dos mecanismos de defesa do hospedeiro. Por outro lado, densidades P. elaeisis acima de 10:1 reduziram a progênie por pupa, o que pode evidenciar interferência mútua entre fêmeas desse parasitoide. A injeção de excesso de partículas semelhantes a vírus, polidnaviroses, que interferem na capacidade de reconhecimento imunológico do hospedeiro (NAKAMATSU; TANAKA, 2003), pode ter causado a morte prematura das pupas de A. gemmatalis, antes que os imaturos de P. elaeisis completassem seu desenvolvimento.

A produção de descendentes por fêmea de $P$. elaeisis teve, também, tendência de aumento, seguida de queda, com maior densidade de fêmeas desse parasitoide, o que seria possivelmente causado por superparasitismo. Isto foi observado para $M$. digitata e Melittobia australica Girault, 1912 (Hymenoptera: Eulophidae) com pupas de N. bullata, e A kamali com M. hirsutus (Silva-Torres; MATTHEWs, 2003; SAgarRa et al., 2000). Outra justificativa contrária a anterior, mas possível, seria quefêmeas desse parasitoide poderiam distinguir hospedeiros parasitados dos não parasitadoseregularem otamanho da postura. Outros estudos mencionam que a competição, tamanho e a idade do parasitoide podem, também, afetar o número de ovos depositados no hospedeiro (Godfray, 1994).

A razão sexual de $P$. elaesis foi superior a $0,90 \mathrm{em}$ todas as densidades. A produção de fêmeas éimportante, pois fêmeas de parasitoides são responsáveis pelo parasitismo e produção de progênie (PEREIRA et al., 2009). Isso foi relatado, também, para esse mesmo parasitoide criado em pupas de B. mori (PEREIRA et al., 2010b) e para Melittobia clavicornis Cameron, 1908, M. australica e M. digitata (Hymenoptera: Eulophidae) em pupas de $N$. bullata, com razão sexual de 0,97; 0,95 a 0,97 e 0,95 a 0,98, respectivamente (GoNZÁLES et al., 2004; SiLva-Torres; MatTHEws, 2003).

Os adultos de $P$. elaeisis foram maiores na densidade de 1:1. Isto éjustificado pela menor competição das larvas por nutrientes e pelo menor número de descendentes por pupa nessa densidade. Independente da densidade, os adultos de P. elaeisis apresentaram tamanho satisfatório, próximo ao encontrado em pupas de B. mori e A. gemmatalis e em T. arnobia após seis gerações nos hospedeiros anteriores (PEREIRA et al., 2010a) e superior quando criados em pupas de B. mori (Pereira et al., 2010b). Isso é importante, pois o tamanho do corpo tem correlação positiva com indicadores de qualidade como longevidade, preferência de cópula, fecundidade, longevidade reprodutiva, emergência da progênie e razão sexual que podemindicar a eficiência de parasitoides. Fêmeas e machos maiores (1,7-2,1 e 1,2-1,6 mm) de A. kamali viveram por mais tempo $(35,4 \pm 10 ; 29,16 \pm 6,5$ dias $)$ do que fêmeas e machos pequenos (1,5-1,9 e 1,0-1,4 mm) $(27,9 \pm 9,6 ; 18,4 \pm 5,7$ dias), respectivamente. Fêmeas de $A$. kamali não mostraram preferência para cópula com machos grandes ou pequenos, no entanto, afecundidadefoi positivamentecorrelacionada como tamanho de fêmeas, sendo de $37 \pm 21$ ovos para fêmeas pequenas e $96 \pm 43$ para as grandes. A capacidade de parasitismo de $A$. kamali, a taxa diária de oviposição e o número de progênie de parasitoides fêmeas dessa espécie foram mais elevadas entre parasitoides grandes. Estas implicações sugerem ser necessário utilizarem-se indivíduos maiores para reprodução, além de acompanhar-se sistematicamente a evolução da criação para assegurar e maximizar a produção massal de parasitoides (SAGARRA et al., 2001).

$P$. elaeisis parasitou e se desenvolveu em pupas de $A$. gemmatalis em todas as densidades testadas demonstrando a adequabilidade do hospedeiro. De maneira geral, a faixa entre seis e oito fêmeas de $P$. elaeisis por pupa de A. gemmatalis foi considerada a mais indicada para a criação desse parasitoide em laboratório, pois proporcionou maior progênie produzida por pupa.

\section{AGRADECIMENTOS}

Ao Conselho Nacional de Desenvolvimento Científico e Tecnológico (CNPq), à Coordenação de Aperfeiçoamento de Pessoal de Nível Superior (CAPES) e à Fundação de Amparo à Pesquisa do Estado de Minas Gerais (FAPEMIG).

\section{REFERÊNCIAS}

ANDRADE, G.S.; SERRAO, J.E.; ZANUNCIO, J.C.; ZANUNCIO, T.V.; LEITE, G.L.D.; POLANCZYK, R.A. Immunity of an alternative host can be overcome by higher densities of its parasitoids Palmistichus elaeisis and Trichospilus diatraeae. Plos One, v.5, p.e13231, 2010.

BARBOSA, L.S.; COURI, M.S.; AGUIAR-COELHO, V.M. Desenvolvimento de Nasonia vitripennis (Walker, 1836) (Hymenoptera: Pteromalidae) em pupas de Cochliomyia macellaria (Fabricius, 1775) (Diptera: Calliphoridae), utilizando diferentes densidades do parasitóide. Biota Neotropica, v.8, n.1, p.49-54, 2008.

BITTENCOURT, M.A.L.; BERTI FILHO, E. Preferência de Palmistichus elaeisis por pupas de diferentes lepidópteros praga. Scientia Agricola, v.56, n.4, p.1281-1283, 1999. 
BITTENCOURT, M.A.L.; BERTI FILHO, E. Exigências térmicas para o desenvolvimento de Palmistichus elaeisis (Hymenoptera, Eulophidae) em pupas de cinco espécies de lepidópteros. Iheringia. Série Zoologia, v. 94, n.3, p.321-323, 2004.

BITTENCOURT, M.A.L.; BERTI FILHO, E. Aspectos biológicos de Palmistichus elaeisis Delvare \& LaSalle (Hymenoptera, Eulophidae) em pupas de diferentes espécies de lepidópteros. Revista de Agricultura, v.81, n.3 p.275-286, 2006.

BITTENCOURT, M.A.L; FARIA, J.C.; BERTI FILHO, E. Influência do sexo e da temperatura, de diferentes espécies de lepidópteros, sobre o parasitismo por Palmistichus elaeisis (Hymenoptera: Eulophidae). Revista de Agricultura, v.79, n.3, p.304-311, 2004.

CHOI, W.I.; YOON, T.J.; RYOO, M.I. Host-size-dependent feeding behaviour and progeny sex ratio of Anisopteromalus calandrae (Hym., Pteromalidae). Journal of Applied Entomology, v.125, n.2, p.71-77, 2001.

CÔNSOLI, F.L.; VINSON, S.B. Clutch size, development and wing morph differentiation of Melittobia digitata Dahms (Hymenoptera: Eulophidae). Entomologia Experimentalis et Applicata, v.102, n.2, p.135-143, 2002.

DELVARE, G.; LASSALE, J. A new genus of Tetrastichinae (Hymenoptera: Eulophidae) from the neotropical region, with the description of a new species parasitica on key pests of oil palm. Journal of Natural History, v.27, n.1, p.435-444, 1993.

GODFRAY, H.C.J. Parasitoids, behavioral and evolutionary ecology. Princeton: Princeton University Press, 1994.

GONZÁLES, J.M.; ABE, J.; MATTHEWS, R.W. Offspring production and development in the parasitoid wasp Melittobia clavicornis (Cameron) (Hymenoptera: Eulophidae) from Japan. Entomological Science, v.7, n.1, p.15-19, 2004.

GREENE, G.L.; LEPPLA, N.C.; DICKERSON, W.A. Velvetbean caterpillar: A rearing procedure and artificial diet. Journal of Economic Entomology, v.69, n.4, p.487-488, 1976.

GUNDUZ, E.A.; GULEL, A. Investigation of fecundity and sex ratio in the parasitoid Bracon hebetor Say (Hymenoptera: Braconidae) in relation to parasitoid age. Turkish Journal of Zoology, v.29, n.1, p.291-294, 2005.

MONTEIRO, L.B.; SOUZA, A.; PASTORI, P.L. Comparação econômica entre controle biológico e químico para o manejo de ácaro-vermelho em macieira. Revista Brasileira de Fruticultura, v.28, n.3, p.514-517, 2006.

NAKAMATSU, Y.; TANAKA, T. Venom of ectoparasitoid Euplectrus sp. near plathypenae (Hymenoptera: Eulophidae) regulates the physiological state of Pseudaletia separata
(Lepidoptera: Noctuidae) host as a food resource. Journal Insect Physiology, v.49, n.2, p.149-159, 2003.

PARRA, J.R.P.; BOTELHO, P.S.M.; CORREAA-FERREIRA, B.S.; BENTO, J.M.S. Controle biológico: uma visão inter e multidisciplinar. In: PARRA, J.R.P.; BOTELHO, P.S.M.; CORREA-FERREIRA, B.S.; BENTO, J.M.S. (Ed.) Controle biológico no Brasil: parasitóides e predadores. São Paulo: Manole, 2002. p.125-137.

PARON, M.R.; BERTI FILHO, E. Capacidade reprodutiva de Trichospilus diatraeae (Hymenoptera: Eulophidae) em pupas de diferentes hospedeiros (Lepidoptera). Scientia Agricola, v.57, n.2, p.355-358, 2000.

PASTORI, P.L.; MONTEIRO, L.B.; BOTTON, M. Biologia e exigências térmicas de Trichogramma pretiosum Riley (Hymenoptera, Trichogrammatidae) “linhagem bonagota" criado em ovos de Bonagota salubricola (Meyrick) (Lepidoptera, Tortricidae). Revista Brasileira de Entomologia, v.52, n.3, p.472-476, 2008.

PEREIRA, F.F.; ZANUNCIO, J.C. Palmistichus elaeisis, uma alternativa de controle para a lagarta parda do eucalipto. Manejo Integrado de Plagas y Agroecologia, v.76, n.1, p.89-91, 2005.

PEREIRA, F.F.; ZANUNCIO, T.V.; ZANUNCIO, J.C.; PRATISSOLI, D.; TAVARES, M.T. Species of Lepidoptera defoliators of eucalypt as new hosts for the polyphagous parasitoid Palmistichus elaeisis (Hymenoptera: Eulophidae). Brazilian Archives of Biology and Technology, v.51, n.2, p.259-262, 2008.

PEREIRA, F.F.; ZANUNCIO, J.C.; SERRÃO, J.E.; PASTORI, P.L.; RAMALHO, F.S. Reproductive performance of Palmistichus elaeisis (Hymenoptera; Eulophidae) with previously refrigerated pupae of Bombyx mori (Lepidoptera; Bombycidae). Brazilian Journal of Biology, v.69, n.3, p.865869, 2009.

PEREIRA, F.F.; ZANUNCIO, J.C.; PASTORI, P.L.; CHICHERA, R.A.; ANDRADE, G.S.; SERRÃO, J.E. Reproductive biology of Palmistichus elaeisis (Hymenoptera: Eulophidae) with alternative and natural hosts. Zoologia, v.27, n.6, p.887-891, 2010a.

PEREIRA, F.F.; ZANUNCIO, J.C.; SERRAO, J.E.; ZANUNCIO, T.V.; PRATISSOLI, D.; PASTORI, P.L. The density of females of Palmistichus elaeisis Delvare and LaSalle (Hymenoptera: Eulophidae) affects their reproductive performance on pupae of Bombyx mori $\mathrm{L}$. (Lepidoptera: Bombycidae). Anais da Academia Brasileira de Ciências, v.82, n.2, p.323-331, 2010 b.

SAGARRA, L.A.; VICENT, C.; STEWART, R.K. Mutual interference among female Anagyrus kamali Moursi (Hymenoptera: Encyrtidae) and its impact on fecundity, progeny production and sex ratio. Biocontrol Science Technology, v.10, n.3, p.239-244, 2000. 
SAGARRA, L.A.; VICENT, C.; STEWART, R.K. Body size as an indicator of parasitoid quality in male and female Anagyrus kamali (Hymenoptera: Encyrtidae). Bulletin of Entomological Research, v.91, n.5, p.363-367, 2001.

SAMPAIO, M.V.; BUENO, V.H.P.; MALUF, R.P. Parasitismo de Aphidius colemani Viereck (Hymenoptera: Aphidiidae) em diferentes densidades de Myzus persicae (Sulzer) (Hemiptera: Aphididae). Neotropical Entomology, v.30, n.1, p.81-87, 2001.

SCHMID-HEMPEL, P. Evolutionary ecology of insect immune defenses. Annual Review of Entomology, v.50, p.529-551, 2005.

SILVA-TORRES, C.S.A.; MATTHEWS, R.W. Development of Melittobia australica Girault and M. digitata Dahms (Parker) (Hymenoptera: Eulophidae) parasiting Neobellieria bullata (Parker) (Diptera: Sarcophagidae) puparia. Neotropical Entomology, v.32, n.4, p.645-651, 2003.
VINSON, S.B.; IWANTSCH. Host suitability for insect parasitoids. Annual Review of Entomology, v.25, p.397419, 1980.

ZANUNCIO, J.C.; TORRES, J.B.; SEDIYAMA, C.A.Z.; PEREIRA, F.F.; PASTORI, P.L.; WERMELINGER, E.D.; RAMALHO, F.S. Mortality of the defoliator Euselasia eucerus (Lepidoptera: Riodinidae) by biotic factors in an Eucalyptus urophylla plantation in Minas Gerais State, Brazil. Anais da Academia Brasileira de Ciências, v.81, n.1, p.61-66, 2009.

ZANUNCIO, A.J.V.; PASTORI, P.L.; KIRKENDALL, L.R.; LINO-NETO, J.; SERRAO, J.E.; ZANUNCIO, J.C. Megaplatypus mutatus (Chapuis) (Coleoptera: Curculionidae: Platypodinae) attacks hybrid Eucalyptus L'Héritier de Brutelle clones in Southern Espírito Santo, Brazil. The Coleopterists Bulletin, v.64, n.1, p.81-83, 2010.

Recebido em $11 / 8 / 11$

Aceito em 19/10/12 